\title{
Perfil del consumidor de suplementos nutricionales de las sedes de los gimnasios de la USTA en Bogotá*
}

\author{
Johann Sebastián Duarte Gómez**
}

\author{
Recibido: diciembre 13 del 2017 • Aceptado: mayo 7 del 2018
}

\section{Resumen}

En Colombia existe un creciente interés por consumir suplementos, sin conocimiento de sus efectos; por ello, es indispensable conocer el perfil del consumidor que asiste a los gimnasios de la USTA en Bogotá. El objetivo fue determinar el perfil del consumidor de suplementos nutricionales en edades de 15 a 35 años en los gimnasios de las sedes de la USTA, a través de la aplicación de un cuestionario de 19 preguntas abiertas y cerradas a 100 voluntarios hombres y mujeres, asistentes a estos gimnasios. Se utilizó un método cuantitativo. Como resultado se caracterizó el uso de la suplementación en sedes de gimnasios de la USTA, confirmándose que los hombres consumen más suplementos prefiriendo batidos proteicos para aumentar su masa muscular. Adicionalmente, se determinó que las mujeres consumen principalmente vitaminas y agentes lipolíticos para disminución de masa grasa. Por último, se identificó que un alto porcentaje cree que el consumo de suplementos les ayuda a cumplir sus objetivos. Se concluyó que un

"Artículo de investigación, vinculado al proyecto de investigación Conocimientos, imaginarios, prácticas y motivaciones frente al consumo de sustancias ergogénicas en estudiantes de cultura física, deporte y recreación de la universidad santo tomás de la Facultad de Cultura Física Deporte y Recreación de la Universidad Santo Tomás, con fianaciación propia. Citar como: Duarte, J. (2018). Perfil del consumidor de suplementos nutricionales de las sedes de los gimnasios de la USTA en Bogotá. Cuerpo, Cultura y Movimiento, 8(2), 67-82. DOI: https:// doi.org/10.15332/2422474X/5118

** Profesional en Cultura Física, Deporte y Recreación. Egresado de la Universidad Santo Tomás, Bogotá, Colombia. Correo electrónico: johannduarte@usantotomas.edu.co 
número considerable de personas que asiste regularmente a gimnasios consume suplementos sin orientación especializada y sin tener que utilizarlos realmente, pero es necesario regular este uso irracional y potencialmente inseguro de los suplementos nutricionales en mejora del rendimiento físico.

Palabras clave: caracterización del perfil, ergogénicos, factores socioeconómicos, nutrición. 


\section{Consumer profile of nutritional supplements from the gyms USTA's seats in Bogotá}

\section{Abstract}

In Colombia there is a growing interest in consuming supplements, without knowledge of their effects; Therefore, it is essential to know the profile of the consumer who attends the USTA gyms in Bogotá. The objective was to determine the profile of the consumer of nutritional supplements in ages of 15 to 35 years in the gyms of the offices of the USTA, through the application of a questionnaire of 19 open and closed questions to 100 male and female volunteers, attendees to these gyms. A quantitative method was used. As a result, the use of supplementation in gymnasiums of the USTA was characterized; confirming that men consume more supplements preferring protein shakes to increase their muscle mass. Additionally, it was determined that women consume mainly vitamins and lipolytic agents to decrease fat mass. Finally, it was identified that a high percentage believe that the consumption of supplements helps them to achieve their objectives. It was concluded that a considerable number of people who regularly attend gyms consume supplements without specialized guidance and without having to actually use them, but it is necessary to regulate this irrational and potentially unsafe use of nutritional supplements in improving physical performance.

Keywords: profile characterization, ergogenic, socioeconomic factors, nutrition, supplements. 


\section{Introducción}

Para poder desarrollar esta investigación se trabajó de la mano con el semillero de Estudios Sociales del Cuerpo y el Movimiento, con la intención de caracterizar a la población que usa suplementos ergogénicos a la hora de hacer ejercicio. Por este motivo, se decidió tomar como población la comunidad académica de la Universidad Santo Tomás de Aquino (USTA), y como primera medida se tuvo en cuenta una muestra de 94 personas en las 4 sedes de la institución, especialmente San Alberto Magno, Central y Angélico.

En consideración con la investigación se expuso de forma breve qué tipos de suplementos existen y cuál es su utilidad específica; esto con la intención de comprender mejor el uso de estos suplementos con las rutinas que se implementan en los gimnasios, las personas que asisten, y también hay que tener en cuenta, el uso indebido de las mismas, con la intención de que en un futuro se pueda implementar un bienestar optimo en calidad de vida, deporte y salud en las personas de la comunidad USTA.

Para lo anterior, el primer objetivo fue caracterizar el perfil del consumidor de suplementos nutricionales en edades de 15 a 80 años que asiste a los gimnasios de las sedes de la Universidad; para poder así de esta manera identificar las preferencias de suplementos nutricionales dependiendo del género, establecer el rango de edad de consumo de suplementos nutricionales de acuerdo a las preferencias y describir los principales criterios que los usuarios de gimnasio tienen para usar los suplementos nutricionales.

Por consiguiente, el planteamiento del problema abordó un análisis sobre uno de los temas que más polémica ha causado en la actualidad: el creciente interés de las personas en llevar una vida fitness y proactiva, por lo anterior creció el uso de productos que optimizan estas necesidades. Jorquera, Rodríguez, Torrealba, Campos y Gracia (2016) argumentan que "no hace más de 10 años las personas consideraban los hábitos de ejercicio únicamente oportunos para un cuerpo ideal de verano, personas dedicadas al fisiculturismo o al cuidado de la imagen publicitaria" (p. 100). La creación de un estilo de vida fitness y el desarrollo de una figura estética más cercana a lo que se ve en una revista publicitaria, trajo consigo una preocupación por mejorar la imagen personal, para de esta manera concientizar en la actualidad a las personas de vivir con hábitos saludables y un encuentro más cercano con la naturaleza, fuera de la oficina. Pero al ser un nicho 
comercial, las empresas comenzaron a generar un mercado, conocido como venta de suplementos ergogénicos.

El artículo de Jorquera et al. (2016) fue una herramienta valiosa para desarrollar una investigación en Colombia, ya que por fronteras culturales o una evidente carencia de investigación sobre el tema no se ha realizado un trabajo investigativo sobre el efecto, uso y consumo de suplementos ergogénicos en el país. Por esta razón, se comenzó a formular una serie de preguntas que ayudaron a entender cómo se lleva a cabo el control del mercado de estos productos; y desde este punto se abordaron un amplio número de inquietudes sobre el tema.

En primer lugar, se quiso saber qué entendían las personas sobre la suplementación; por ejemplo, si conocían las diferentes presentaciones de estos productos, las utilidades que tienen y cómo distinguirlas. Juhn M. (2003) dice que

[...] estos productos están hechos a base de vitaminas, minerales, materiales de hierbas o plantas, se pueden encontrar en diferentes presentaciones como píldoras, capsulas, tabletas o en forma líquida y usados como suplemento dietario, pero no deberían ser considerados un sustituto para la comida. (P. 922).

En segundo lugar, existe una peculiaridad en la población, siendo los estudiantes adolescentes los que con mayor frecuencia consumen suplementos nutricionales. Colls, Gómez, Cañadas y Fernández (2015) afirman que: "presentan menores conocimientos de las instrucciones de uso de estos productos y son usuarios que hacen actividad física con una intensidad de más de 4 días a la semana (...). Por lo tanto, necesitan un régimen dietario alto" (p. 838). Por este motivo, la utilización de estos suplementos se justifica por las funciones que le atribuyen a la composición corporal, con el aumento de masa magra o disminuyendo el porcentaje graso en un sujeto que orienta sus objetivos a lograr resultados a corto plazo. Por lo tanto, es necesario adoptar medidas que permitan controlar el uso irracional e inseguro de los suplementos nutricionales con la intención de mejorar el rendimiento físico, la salud o la estética.

En tercer lugar, se encuentra el mercado de suplementos y ergogénicos en Colombia que, por su alto valor, genera una variedad infinita de productos y como resultado, de discusiones entre los consumidores. Dado 
que la gran mayoría de los productos suplementarios son norteamericanos, se incrementa su costo y el valor de venta para el consumidor final, lo que fortalece la aceptación de consejos por terceros para el uso de suplementos no aptos para la morfología o para los objetivos que busca el cliente. Bishop (2010) refiere que, por esto, las personas desconocen los productos certificados, naturales e ilegales que pueden encontrarse en el mercado, dado que falta control legal sobre la venta de productos suplementarios y sobre los organismos o personas que deben distribuirlos. Esta última parte ha fundamentado las evidencias múltiples y las frecuentes incongruencias en el uso de estos suplementos, con relación a las razones que justifiquen su uso.

\section{Métodos}

La muestra estuvo compuesta por un grupo no aleatorio de 94 sujetos asistentes a los gimnasios de las sedes de la Universidad Santo Tomás de la ciudad de Bogotá D. C. Los sujetos considerados fueron aquellos que, al momento de realizar el estudio, estaban asistiendo sin interrupción al gimnasio y que voluntariamente aceptaron participar. El instrumento usado fue un cuestionario estructurado de 17 preguntas. Se aplicó a modo de encuesta (pregunta-respuesta) y se realizó en formato "autocompletado" vía virtual.

El tipo de enfoque fue cuantitativo, porque se pretendió encontrar el perfil del consumidor de suplementos nutricionales para identificar las preferencias de los usuarios, por esta razón se obtuvo que este estudio en la recolección de datos arrojó una representación numérica que fue analizada desde los métodos estadísticos según Hernández, Fernández y Baptista (2006).

Por otro lado, el tipo de alcance fue descriptivo, ya que buscó especificar las características, propiedades y perfiles de un grupo poblacional que en este caso fueron personas que asistían a los gimnasios de la Universidad Santo Tomás entre 3 y 6 días a la semana y que consumían algún tipo de suplementación nutricional, lo que quiere decir que se pretendió estudiar cada uno de los componentes de las variables de manera independiente, con el fin de interpretar los valores estadísticos encontrados en cada variable sin llegarlos a relacionar en algún momento, como lo propone Hernández et al. (2006). 


\section{Procedimiento}

Los criterios que se tuvieron en cuenta en la metodología de investigación fueron: que se perteneciera a la Universidad, que no estuvieran en sedes fuera de Bogotá D. C., que entrenaran por lo menos 3 veces por semana, que usaran algún tipo de suplementación nutricional, y que fueran mayores de 15 años o menores de 80 años. De esta forma el tipo de diseño fue no experimental transversal, por la estandarización de la población. Así, la manera de generar una recolección de datos fue una encuesta para los fines anteriormente descritos.

Fue indispensable en la investigación planteada, el tratamiento de variables para determinar el tiempo, las horas y días que entrenaban los usuarios, ya que si se consideraba el tiempo en el que el usuario permanecía en el gimnasio, podría existir la necesidad de consumir suplementos nutricionales, ya que habría un mayor interés de alcanzar los objetivos planteados por la persona. Lo anterior fue considerado como otro tipo de variable a estudiar y que nos daría cuenta de tres tipos de variables más, como es el tipo de suplementación utilizada dependiendo del género, que fue importante identificar y de la misma manera fue necesario establecer el rango de edad de consumo de suplementos nutricionales de acuerdo a las preferencias.

\section{Instrumento}

Teniendo en cuenta la metodología aplicada en este proyecto, el instrumento aquí presentado tuvo en cuenta la participación de la comunidad universitaria de forma voluntaria. La Universidad Santo Tomás de Aquino en Bogotá D. C. fue informada sobre este proceso experimental. Además, los investigadores obtuvieron la firma del consentimiento informado de cada una de las personas responsables en esta encuesta.

\section{La encuesta}

Fue diseñada con un cuestionario de 17 preguntas que se aplicó a modo de encuesta (pregunta-respuesta) y se realizó en formato "autocompletado" vía virtual, donde las preguntas utilizadas estaban diseñadas para clasificar al consumidor bajo criterios como el género, rangos de edad, preferencias de 
suplementación, factores socioeconómicos como lo son la capacidad económica para la adquisición de suplementos, nivel de educación, cantidad de tiempo dedicado al ejercicio y preferencias en el entrenamiento, entre otros.

\section{Resultados y discusión}

Debido al tipo de estudio desarrollado, se logró efectuar una muestra de 94 encuestas realizadas principalmente en la sede central y en la sede San Alberto Magno, con una participación del $31.92 \%$, la sede Doctor Angélico con una participación del $35.11 \%$. La sede Aquinate solo generó una muestra, que corresponde al $1.06 \%$ sobre el total de la muestra.

Con lo anterior, se pudieron obtener los siguientes resultados:

Para empezar, se obtuvo una mayor participación de hombres (55.32\%) que de mujeres ( $44.68 \%$ ), donde los encuestados fueron docentes, administrativos, estudiantes y egresados; y al ser un estudio realizado en una universidad, se entiende por qué en la encuesta hubo un $78.723 \%$ de participación de estudiantes. Además, la mayor participación estuvo en estudiantes de entre 21 y 22 años. se resaltan sin embargo, participaciones de personas mayores de 40 años, siendo una persona de 79 años la de mayor edad y moviendo la desviación estándar del estudio a 8.780 recuentos.

Figura 1. ¿Qué nivel de estudio tiene usted?

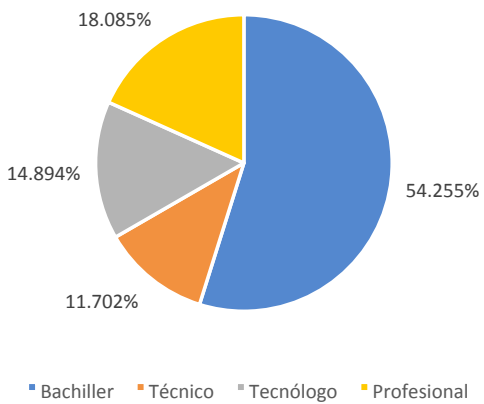

Fuente: elaboración propia. 
La Figura 1 evidencia el nivel de estudio de los encuestados, y los resultados nos resaltan que el $54.255 \%$ de estos son bachilleres, mientras que la muestra de técnicos, tecnólogos y profesionales llega a ser del $44.681 \%$. De hecho, se puede inferir que en esencia la mitad de los encuestados pueden adquirir los suplementos en primera persona, gracias a que tienen un nivel de estudio óptimo para trabajar.

Desde el concepto general del análisis encontramos puentes de relación entre el tiempo de asistencia al gimnasio, los días que asiste al gimnasio a la semana y el tiempo de entrenamiento. Por un lado, la mayoría de los encuestados llevan más de 2 años asistiendo al gimnasio, donde el $58.511 \%$ son personas que llevan asistiendo a este entre 2 y 8 años, Mientras que el $41.489 \%$ tiene una asistencia inferior a un año y el $23.404 \%$ asiste desde hace un año.

Figura 2. ¿Cuántos días a la semana asiste al gimnasio?

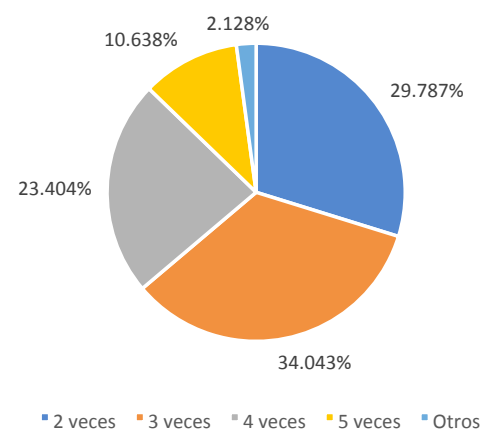

Fuente: elaboración propia.

Por otra parte, se puede apreciar una tendencia decreciente con el número de días de asistencia al gimnasio. En la Figura 2 se puede apreciar que la mayoría de los encuestados practica 3 veces a la semana ejercicio, lo que se puede entender gracias al objetivo de asistir al gimnasio para mejorar la salud, mientras que la disminución de días practicados tiene que ver con los hábitos de ejercicio, con objetivos como tonificación, ganar masa muscular magra, terapia, recreación, gusto y fisiculturismo. Al mismo tiempo, independientemente de la asistencia, el $55.319 \%$ de los encuestados practica 2 
horas de entrenamiento, mientras que el $38.298 \%$ de los encuestados solo practica una hora; completando la participación con 88 encuestados, solo 6 personas realizan más de 2 horas de ejercicio, pero no se tuvo en cuenta el criterio de usar 4 o 5 horas de ejercicio. Relacionando esto con los resultados de la Figura 2, se pueden distinguir dos cosas: primero, las personas que asisten hasta 2 horas al gimnasio lo hacen con la intención de mantener la salud, realizar terapia y mantener un buen estado físico; segundo, algunos de los encuestados creen que con este tiempo de asistencia pueden cumplir con los objetivos de tonificación, masa muscular y entrenamiento competitivo. Por lo dicho, se puede comenzar a cuestionar la motivación de hacer ejercicio con el uso o sin el uso de suplementos ergogénicos, teniendo en cuenta los resultados obtenidos.

Figura 3. ¿Sigue actualmente algún plan de alimentación especial, fuera de dieta normal?

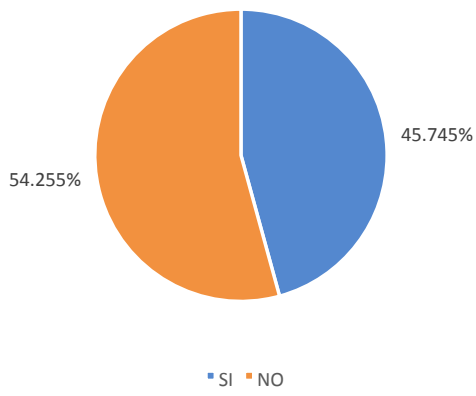

Fuente: elaboración propia.

$\mathrm{Al}$ analizar cuántas personas utilizaban un plan de alimentación adecuado en la Figura 3, se encontró que para esta pregunta el $54.255 \%$ manifestaron que no tenían ningún plan de alimentación, mientras que el $45.745 \%$ si mantenían un plan de alimentación fuera de la dieta normal. Con la intención de analizar más a fondo sobre el plan de alimentación, se preguntó sobre su prescripción, donde se encontró que el $58.511 \%$ de los encuestados se autoprescriben, el $4.255 \%$ por recomendación de amigos, el 
$2.128 \%$ de entrenadores y el $3.191 \%$ de deportólogos; esto nos permite verificar que el $68.085 \%$ de las encuestados continúan utilizando mecanismos de consulta para dieta que no son óptimos por no pertenecer al campo profesional para poder hacerlo, solo el $31.915 \%$ utiliza la recomendación de un nutricionista.

Para complementar el análisis, el siguiente paso fue determinar el uso de suplementos con la respuesta anterior; y se obtuvo que el $71.277 \%$ de la muestra no consumía suplementos, mientras que el $28.723 \%$ sí lo hacía. Pero al corroborar esta información con la recomendación de prescripción del suplemento, el $73.404 \%$ contestó autoprescripción y con ello se pudo determinar que alguna vez en la vida, han comprado y utilizado suplementos, mientras que de estos solo el $7.447 \%$ utilizaba el consejo de un nutricionista. El resto sigue escuchando personas no especializadas que les recomiendan inclusive los suplementos.

En cuanto al espacio de consumo de la suplementación, y pese al énfasis de no consumo de suplementos por parte del $70.213 \%$ de los encuestados, se puede inferir que la mayoría de los consumidores de suplementos lo hacen con objetivos de ganancia de masa muscular y suplemento dietario. De igual manera, se ha determinado consumo para apoyar el rendimiento deportivo y la pérdida de grasa.

Por otro lado, la frecuencia del consumo de suplementos presenta un uso descendente en el número de días; por un lado, el uso más habitual es de 2 veces a la semana con un $10.638 \%$, siendo una vez por semana la menor frecuencia y de 6 a 7 veces por semana la mayor frecuencia. Esta última frecuencia representa a una persona que se dedica al fisiculturismo. Mientras que para el número de veces que se consume estos suplementos al día, el $18.085 \%$ de los encuestados utiliza los suplementos 2 veces al día, donde el mínimo es una vez al día y fue curioso encontrar el máximo de 5 veces al día, identificando a un encuestado que, o se mantiene solo con suplementación o tiene un plan de alimentación significativamente alto en calorías que lo compense en el entrenamiento. 
Figura 4. ¿Considera que un plan alimentario individualizado puede ayudarlo a cumplir sus objetivos, siendo tal vez una mejor alternativa que los suplementos nutricionales?

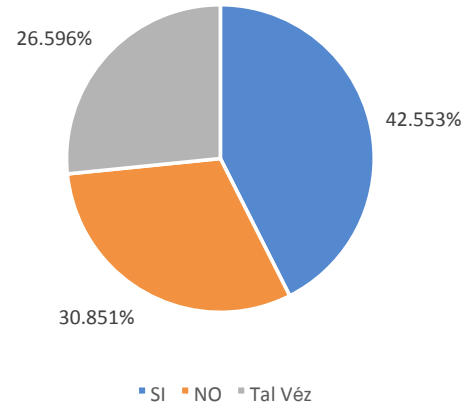

Fuente: elaboración propia.

Por último, se tuvo en consideración si se alcanzaron los efectos esperados con la suplementación y los planes de alimentación, donde se pudo inferir que el $19.149 \%$ de los encuestados que consumen suplementos sí han logrado los efectos esperados, el $2.128 \%$ no ha obtenido los efectos deseados, mientras que el $9.574 \%$ solo ha conseguido los efectos parcialmente. Al sugerir un plan alimenticio como alternativa de los suplementos, la Figura 4 nos indica que el $42.553 \%$ sí considera esta opción; por otro lado, el $30.851 \%$ no lo considera y el $26.596 \%$ sustenta que podría ser así. Permitiéndonos lo anterior inferir que el $69.149 \%$ de los encuestado pueden optar por alternativas alimenticias como ayuda en el ejercicio en lugar de la suplementación.

El perfil del consumidor de suplementos nutricionales que asiste a los gimnasios de la Universidad Santo Tomás en las sedes de Bogotá D. C., está definido por consideraciones frente al entorno sociocultural de la población, sus hábitos de vida y los objetivos que desea alcanzar con su entrenamiento en el gimnasio. En este punto, ya hubo opciones de hablar sobre el tiempo de entrenamiento, el tipo de suplementación que usan y las fuentes de información que utilizan para experimentar estos productos, si es el caso.

La muestra generó una participación significativa entre los rangos de 17 a 79 años; donde la población más activa está entre los 21 y 22 años 
y permitió hablar sobre los estudiantes principalmente. Este estudio no ha sido implementado por otras universidades en el país, por lo que las comparaciones fueron consideradas con estudios previos en otros países, donde se pudieron comparar resultados frente a las poblaciones. El factor que de alguna manera dificultó un poco la comparación era el tamaño de la muestra y que estos estudios fueron diseñados para poblaciones más grandes, como es el caso del trabajo de Jorquera et al. (2016) en gimnasios de Santiago de Chile.

Países como Chile, Brasil, España y otros, presentan un consumo significativo de suplementos por parte de las personas que realizan entrenamiento en los gimnasios, mientras que los estudiantes de la Universidad Santo Tomás de Aquino, en un $70.21 \%$, no consumen suplementos; esto en cierta medida puede significar que al ser una muestra conformada principalmente por estudiantes, muchos de ellos no cuenten con recursos para adquirirlos, notándose especialmente de que un poco más de la mitad de los encuestados son bachilleres; como también, que estos han intentado averiguar sobre la suplementación a través de mecanismos de consulta que no son óptimos.

Por otro lado, no se pudo confirmar que los hombres sean mayores consumidores que las mujeres, ya que en la muestra solo por un $5 \%$ se puede apreciar esta razón. Además, sí se lograron detallar varios aspectos frente a las opciones del consumo de suplementos nutricionales: mientras los hombres optan especialmente por productos para el aumento magro y la tonificación, las mujeres prefieren los de pérdida de grasa y componente dietario.

Con lo anterior, se puede ver que los estudiantes de La Universidad Santo Tomás de Aquino no presentan en la mayoría un uso que necesite una intervención; sin embargo, las personas que usan suplementación usan medios de información que no son óptimos y no se atienen a una dieta adecuada. Al tener una de las mismas posturas de cuestionamiento que los trabajos realizados en el resto del mundo, utilizar una suplementación debe ser un objetivo direccionado por un equipo de profesionales que pueda facilitar el cumplimiento de las metas personales de cada participante, por ello es fundamental caracterizar el perfil del consumidor.

Esto es importante puesto que gestiona vías de investigación, solución y tratamiento en el uso de suplementos nutricionales que no sean favorables para cada persona. Además, a largo plazo esta acción podría poner en el 
mercado empresas poco éticas que impulsen el consumo de suplementos y que en el tema publicitario, pongan en riesgo la vida, el bienestar de las personas y el desarrollo del mercado. Grandes potencias como EE. UU. o Gran Bretaña ya están interviniendo este mercado, debido a las coyunturas sociales que pueden generarse, si productos poco reglamentados llegan a afectar a personas y familias.

\section{Conclusiones}

El perfil del consumidor de suplementos que se encontró en las sedes de los gimnasios de la Universidad Santo Tomás de Aquino, en los distintos géneros masculino y femenino, nos permitió evidenciar que los jóvenes son conscientes de que una alimentación saludable está por encima de una suplementación adicional, para poder generar una nueva hipótesis que nos lleve a indagar sobre nuevas variables de investigación que surgen, como son, si la carrera profesional incide en la decisión de no usar algún tipo de suplementos nutricionales o si de esta manera se puede mantener un cuerpo saludable.

Gracias a que el estudio se realizó en una universidad, que la encuesta fue direccionada principalmente por estudiantes y que hubo participación principalmente de la sede central, la sede San Alberto Magno y la sede Doctor Angélico, el rango de edad con mayor significancia del estudio estuvo entre los 21 y los 22 años. Esto permite establecer un análisis sobre la caracterización de la suplementación entre los 17 y los 79 años..

La investigación en sus resultados mostró que tanto hombres como mujeres en estas sedes no tienen preferencias por la suplementación nutricional en gran volumen, lo que significa que no siempre las horas de entrenamiento y los objetivos que se desean obtener en el gimnasio están directamente relacionados con suplementos que aceleren los procesos estéticos del cuerpo, con el fin de aumentar la masa muscular o disminuir el porcentaje graso, por esta razón la dieta se vuelve indispensable para lograr objetivos sin importar el tiempo que se tarde en su efectividad en comparación con la suplementación.

Al analizar las características del uso de suplementos por características generales del individuo frente a su estilo de vida, se pueden destacar tres cosas: primero, sin importar si es docente, administrativo, estudiante o 
egresado, las persona utiliza entre 1 a 2 horas para entrenar; segundo, todos los participantes tienen un interés en la práctica del gimnasio con diferentes propósitos; y tercero, la mayoría de las personas han practicado entre 2 y 8 años, con una relación entre los días practicados; es decir, el principal objetivo es mejorar la salud, mientras que la disminución de días practicados tiene que ver con los hábitos de ejercicio, con objetivos como tonificación, ganar masa magra, terapia, recreación, gusto y fisiculturismo.

Al determinar los hábitos más característicos para la selección y uso de suplementos nutricionales gracias a las expectativas, valores y creencias más destacadas de los encuestados, se enfatiza que aquellos que asisten hasta 2 horas al gimnasio lo hacen con la intención de mantener la salud, realizar terapia y mantener un buen estado físico; algunos de los encuestados creen que con este tiempo pueden cumplir con los objetivos de tonificación, masa muscular y entrenamiento competitivo. Prefieren utilizar un plan de alimentación antes que la suplementación, pese que en algún momento pudieron haber intentado consumirlos por alguno de los objetivos anteriormente expuestos.

Por otro lado, también se ha visto que los encuestados continúan utilizando mecanismos de consulta para dieta o suplementación que no son óptimos, ya que no pertenecen al campo profesional para poder hacerlo. Además de que el $69.149 \%$ de los encuestados puede optar por alternativas alimenticias como ayuda en el ejercicio en lugar de la suplementación para perdida de grasa, ganancia muscular, suplemento dietario y un motor de ayuda para las sintomatologías propias de la tercera edad.

Como se ha descrito anteriormente, se puede concluir que los principales criterios que los usuarios de los gimnasios de la Universidad Santo Tomás tienen para usar los suplementos nutricionales, pese a que la mayoría de los encuestados no usan suplementos, son para ayudar a la perdida de grasa, aumento muscular y síntomas de la tercera edad; con lo cual dentro de las preferencias de consumo están: las aminoácidos, suplementos dietarios, mejoras de rendimiento, proteínas, quemadores de grasa, vitaminas y Ensure.

Finalmente, el perfil del consumidor de suplementos de las sedes de la Universidad Santo Tomás de Aquino, debido a su participación casi igual en hombres y en mujeres, no tuvo en cuenta este objetivo; al ser los estudiantes la mayor participación de la encuesta, esto influyó para determinar que las 
personas de la muestra son más conscientes sobre el uso de la suplementación y los planes de alimentación, pese a que todavía no utilizan canales de información y prescripción óptimos para acompañar a su actividad física, teniendo en cuenta que la mayoría se ejercita 3 veces a la semana y de 1 a 2 horas diarias, con el objetivo de mejorar su rendimiento deportivo, perder grasa, aumentar masa muscular magra o mejorar su salud.

\section{Referencias}

Bishop, D. (2010). Dietary supplements and team-sport performance. Sports Medicine, 40(12),995-1017.DOI: https://doi.org/10.2165/11536870-000000000-00000.

Colls Garrido, C., Gómez, J. L., Cañadas-De la Fuente, G. y Fernández-Castillo, R. (2015). Use, effects, and knowledge of the nutritional supplements for the sport in university students. Nutrition hospitalaria, 32(2), 837. Recuperado de http://www.ncbi.nlm.nih.gov/pubmed/26268119

Hernández Sampieri, R., Fernández Collado, C. y Baptista Lucio, M, P. (2006). Metodología de la investigación. México D. F., México: McGraw-Hill/ Interamericana Editores S. A. de C. V.

Jorquera, C., Rodríguez, F., Torrealba, M. I., Campos, J. y Gracia, N. (2016). Consumo, características y perfil del consumidor de suplementos nutricionales en gimnasios de Santiago de Chile. Revista Andaluza de Medicina del Deporte, 9(3), 99-104. DOI: https://doi.org/10.1016/j.ramd.2015.04.004.

Juhn, M. S. (2003). Popular sports supplements and ergogenic aids. Cham: Adis International. DOI: https://doi.org/10.2165/00007256-200333120-00004 